\title{
Methodological approaches of mathematics intersubject training with the use of computer mathematical packages in the training system of future bachelors and masters of non- mathematical disciplines under the conditions of informatization
}

\author{
Sergey I. Gorlov ${ }^{1}$, Toufic B. Kaziakhmedov ${ }^{1, *}$ \\ ${ }^{1}$ Nizhnevartovsk State University, 628605, 56 Lenina str., Nizhnevartovsk, Russia
}

\begin{abstract}
Quality of mathematical knowledge in the training system of bachelor's and master's of non-mathematical disciplines is a problem of current education. Unfortunately, the newly appeared opportunities of improving the mathematical training quality of the above-mentioned bachelors are not used due to origin of the wealthiest tools of computational and symbolic mathematics. We will think as before: if we have a mathematical thinking, we will get the knowledge. But formation of mathematical thinking of the whole professional community representatives is not only feasible, but also economically profitable, and there is no need for this as well. The use of mathematical methods in various areas of professional activity under the conditions of implementation of information technologies into all spheres of human activity requires revision of teaching methods, content of mathematical training, study of computational methods and mathematical simulation tools.

This article focuses on innovative approaches to mathematical training of bachelors and masters of non-mathematical areas associated with the use of mathematics as a tool for solving professional problems.
\end{abstract}

\section{Introduction}

In the age of information and emergence of a plurality of computer packages and mathematic simulation packages it is required to change both the content and methodological approaches of teaching mathematics. This conclusion is based on long-term observations of bachelors' attitude toward mathematics when graduating from a higher education establishment and immediately after bachelors' employment as well as in the course of the work. The following parameters were being analyzed: mathematical disciplines in bachelor's and master's plans, mathematical methods in the field of future

*Corresponding author: ktofik@yandex.ru 
profession, quality of mathematical knowledge, and understanding the need for the use of computer mathematical packages by a graduate.

It was assumed at the beginning of informatization that the mathematics would become not only a lot of chosen people, but also of the broad segments of professional community. It is obvious that there is no any professional activity area, where one can do without mathematical methods of analysis and measurement (calculations). But all the same, the majority of graduates of non-mathematical disciplines do not see the importance of mathematical knowledge in their professional activity. It is because the approach of teaching mathematics itself contributes to such a vision of mathematics role above all things. Firstly, we are trying to give them the whole science invariant covering the largest possible number of sections without reference to future professional activity by inertia and with the decrease in the number of real hours concerning mathematics learning. Then, we want to teach mathematical methods and computer math packages by means of optional courses. At the same time, we come across the fact that they have not mastered the mathematics for some reason. Let us present our observations and results of the answers to some of our questions to graduates at graduation and workplaces.

"The approach focused on the study of mathematics invariant as a general course and then adapting mathematics to professional field does not always prove itself specifically in training of bachelors and masters of non-mathematical areas." [3]

The purpose of the study is to identify the factors contributing to the change of attitude toward mathematics as a toolkit for solving job tasks.

In much the same way as information technologies have radically changed the professions existing over the course of decades in the economic sphere, the abovementioned also contribute to change in the disciplines being taught at universities and colleges toward metadisciplinarity (inter-disciplinarity), especially in the field of mathematics study by bachelors and masters of non-mathematical areas in the same way. This is a research hypothesis. Thus, it is necessary to determine new content-related and methodological approaches for formation of knowledge from the field of mathematics for use in professional activity.

\section{Materials and Methods}

The following parameters as progress in mathematics, understanding the need of mathematical knowledge in the professional field were being considered as part of the study. Such methods as analysis, questionnaire survey, and practical testing the skills of the use of mathematical packages in problem solving were being used.

In recent years, final test acts as a form of control in non-mathematical training profiles in the Russian higher education, so we considered those areas, wherein an exam or a grading test in mathematics is being taken. Academic records are presented in the following table.

Table 1. Approximate academic progress of graduates in mathematics.

\begin{tabular}{|l|c|c|c|}
\hline Department & $\begin{array}{c}\text { Number of } \\
\text { students }\end{array}$ & $\begin{array}{c}\text { Marks "4" and } \\
\text { "5" }\end{array}$ & Mark “3" \\
\hline Department of Humanities & 30 & 4 & 26 \\
\hline $\begin{array}{l}\text { Department of Pedagogics and } \\
\text { Psychology }\end{array}$ & 40 & 22 & 18 \\
\hline Graphic Arts Department & 20 & 11 & 9 \\
\hline Physical Education Department & 30 & 6 & 24 \\
\hline Faculty of Economics & 40 & 14 & 26 \\
\hline Total: & 160 & 57 & 103 \\
\hline
\end{tabular}


The same students were asked to answer the following questions:

Do you need math in the future professional activity? (If yes, exactly what kind of branches of mathematics?)

Are you familiar with mathematical computing packages? (If yes, list them)

Are you familiar with computer programs designed for your professional activity only? (Please, list them).

Table 2. Results of students questioning.

\begin{tabular}{|c|c|c|c|c|c|c|c|c|}
\hline \multirow[t]{2}{*}{ No. } & \multirow[t]{2}{*}{ Department } & \multirow[t]{2}{*}{ 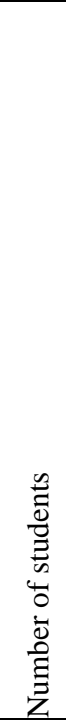 } & \multicolumn{2}{|c|}{ 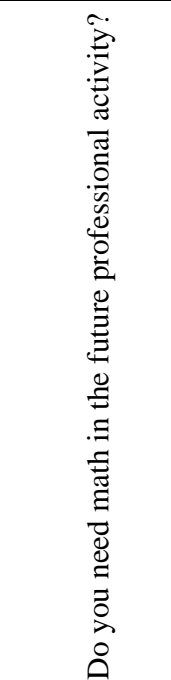 } & \multicolumn{2}{|c|}{ 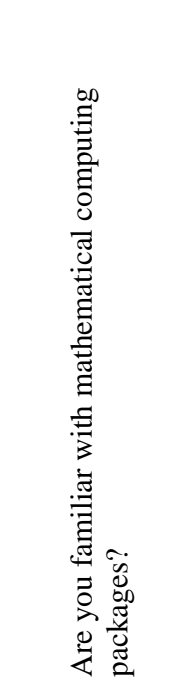 } & \multicolumn{2}{|c|}{ 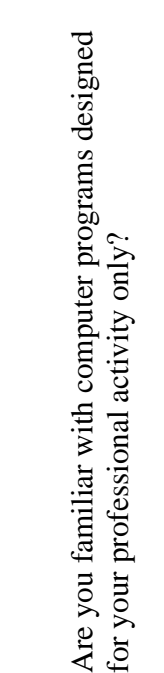 } \\
\hline & & & Yes & No & Yes & No & Yes & $\mathrm{No}$ \\
\hline 1 & $\begin{array}{l}\text { Department of } \\
\text { Humanities }\end{array}$ & 30 & 4 & 26 & 26 & 4 & 26 & 4 \\
\hline 2 & $\begin{array}{l}\text { Department of } \\
\text { Pedagogics and } \\
\text { Psychology }\end{array}$ & 40 & 2 & 38 & 26 & 14 & 26 & 14 \\
\hline 3 & Graphic Arts Department & 20 & 1 & 19 & 11 & 9 & 11 & 9 \\
\hline 4 & $\begin{array}{l}\text { Physical Education } \\
\text { Department }\end{array}$ & 30 & 6 & 24 & 6 & 24 & 6 & 24 \\
\hline 5 & Faculty of Economics & 40 & 14 & 26 & 40 & 0 & 40 & 0 \\
\hline & Total & 160 & 27 & 133 & 109 & 51 & 109 & 51 \\
\hline
\end{tabular}

Regarding the latter issue, the students mainly listed the technologies, which they learned in the course of "Informatics" or "Information Technologies" discipline with the exception of students of economic trends. They noted the importance of knowing the different modules of 1C package. Packages of mathematical calculations are little known to them. This question conveys the suggestion that there is no approach of teaching mathematics based on the capability of these packages with a focus on the area of professional activity.

Improving the quality of teaching mathematics is a problem being studied both in school and university education $[4,5,6]$.

Changes that occur in the extent of academic load toward reduction in mathematical disciplines for bachelors of non-mathematical profiles are perceived by teachers with incomprehension. The dissatisfaction is based on the following understanding: it is necessary to possess mathematical thinking [7] in order to calculate and simulate.

It is necessary to clearly determine which mathematical background is relevant to this area, and which computational tools they should know. We come to the conclusion that it is 
necessary to pass from mathematical thinking formation task to practice of mathematical calculations and simulation in these areas, while such sections as the theory of probability and mathematical statistics, computational mathematics (Numerical methods for mathematicians), methods of optimal decisions (optimization methods for mathematicians), computational geometry, management theory, mathematical methods in the subject area (e.g., mathematical methods in economics, sociology, psychology, etc.) shall be included in the concept of computational science. We think that the mathematics invariant should be studied in the course of studying the discipline on the intersubject basis, for example "Mathematical methods in professional activity."

Western scientists have recently been allocating a computer science in a separate subject area. We will not analyze the justifiability of this approach, but we must bear in mind that the wealthiest computer packages of computer programs have been developed by mathematicians and programmers. If we basically mean computational mathematical methods and a set of developed computational tools by computer science then we do not see any contradiction here. For example, the Advisory Committee for Information Technologies at the President of the United States presented an analytical report regarding this issue in 2005. The report contains the analysis results of the state of things regarding development of informatics in the United States and it is shown that there is a need to take decisive actions here at the federal level in order to prevent those negative trends currently observed both in American science, and in the education system. It is shown in the report that the development of "computer science" creates the unique opportunities for conducting of research studies today [1]. We just want to add that the existing computer mathematical packages have unique didactical tools of improving the quality of mathematical education.

As for actual computational aspects of informatics, they have always been given a considerable attention in Russia. In this case, a scientific field was formed, which has been actively developed by Russian scientists for more than 35 years already and has got the name of computational experiment in our country. The academician A. A. Samarsky is the founder of this field.

Computational experiment is a method of studying facilities or physical processes by means of mathematic simulation. This method assumes that following the construction of a mathematical model its numerical study making it possible to "play" the behavior of the object under study under different conditions or in various versions is conducted [2].

Thus, information technologies should promote the use of mathematical apparatus in all spheres of economic activity, wherein calculations and mathematical assessment of various parameters of economic management is needed.

First of all, we shall note that the mathematical packages contribute to a formalized perception of the problem formal structure, formation of logical thinking in the sphere of quantitative and spatial relationship, mathematical memory as well as culture of mathematical calculations.

Mathematical packages make it possible to visualize the basic concepts and even conduct the experiments defining the behavioral essence of functions, predicates and methods at the study level.

For example, when studying the bases of mathematical logic, the mathematical packages enable not only to learn and remember the basic functions, but also to test them at work via changing the parameter values. Logical functions in EXCEL are shown in Fig. 1. 


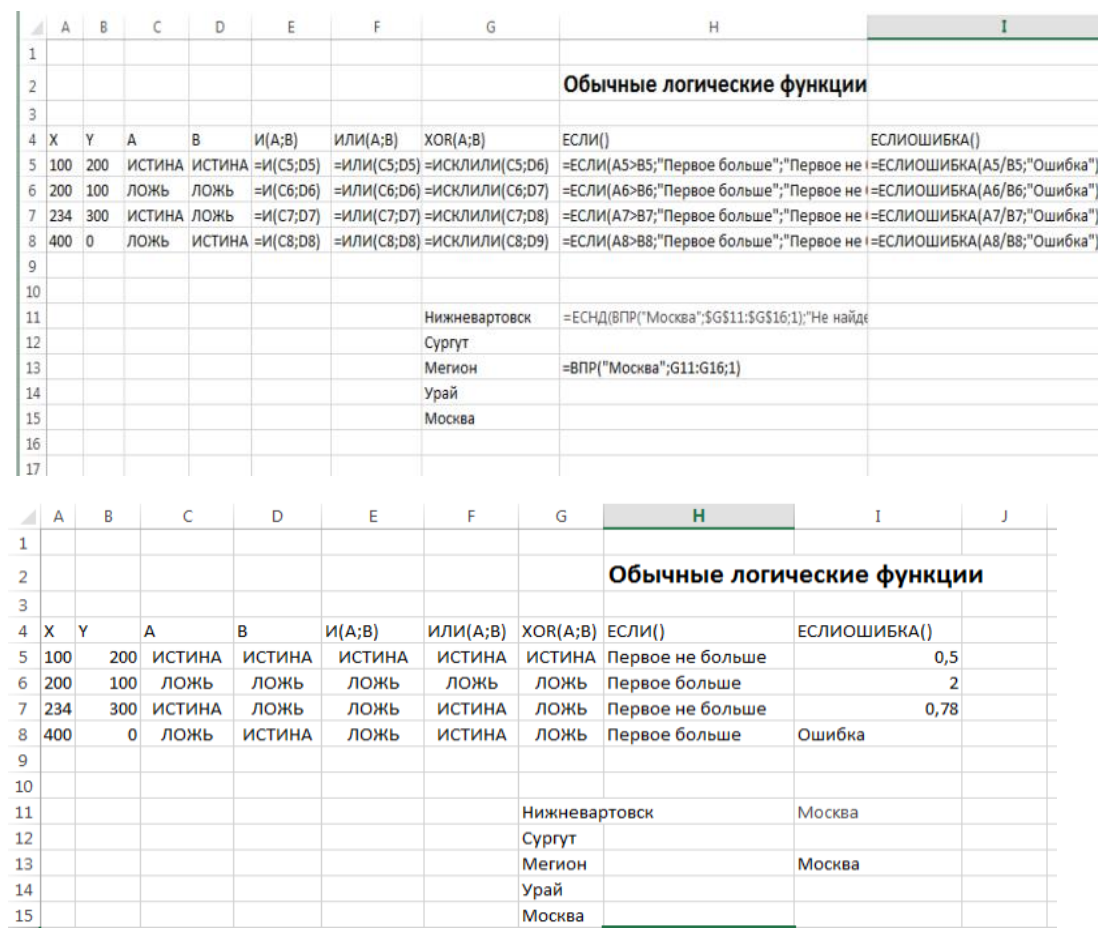

Fig. 1. Example of logical functions use

Besides, these functions are used in the simplest problems of a professional field.

Mathematical software packages play a very important role in the formation of logical thinking capability. Let us consider the simplest tables of calculating a definite integral.

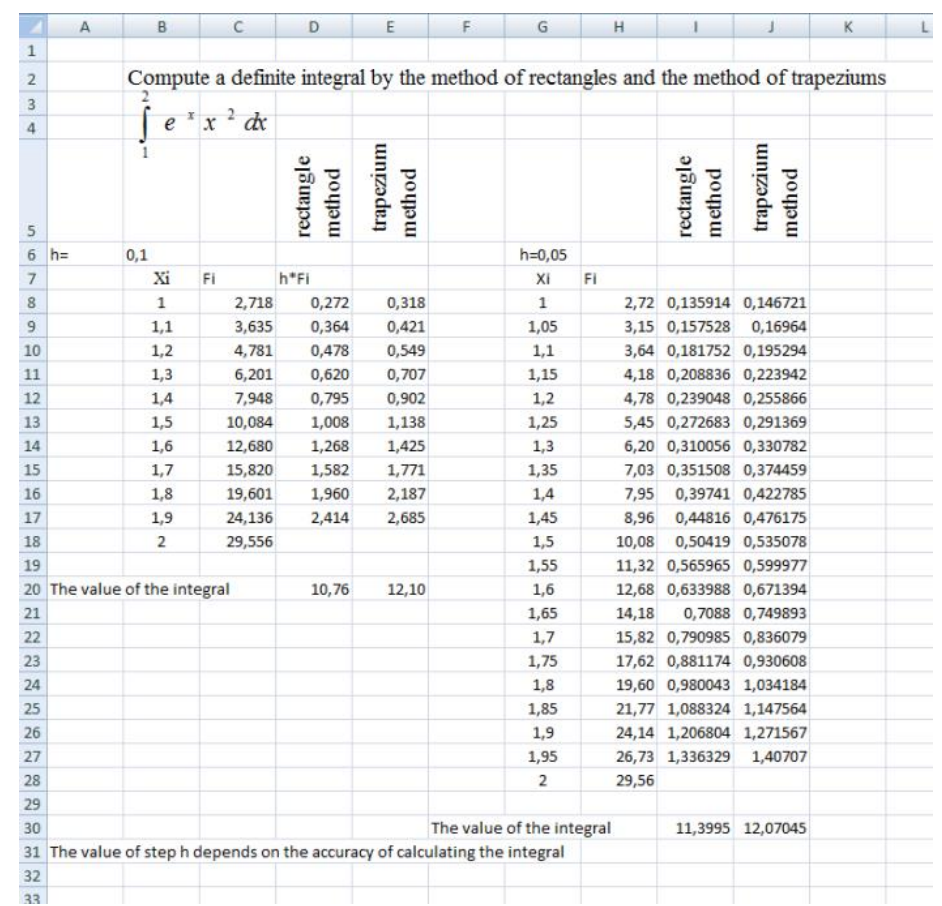

Fig. 2. Evaluation of the definite integral via various methods. 
For more details of using EXCEL as a tool for formation of quality mathematical knowledge and skills refer to [3].

Bringing professional tasks to problems of functional analysis, matrix algebra, computational tasks as well as statistics and geometry problems is very important, but also the most difficult part of forming the quality of mathematical competencies in professional activity of bachelors and masters of humanitarian trends.

Attempts to study these sections in the subject "Mathematics" and then demonstrate that here they say mathematics is a serious tool for your profession and does not produce the desired results.

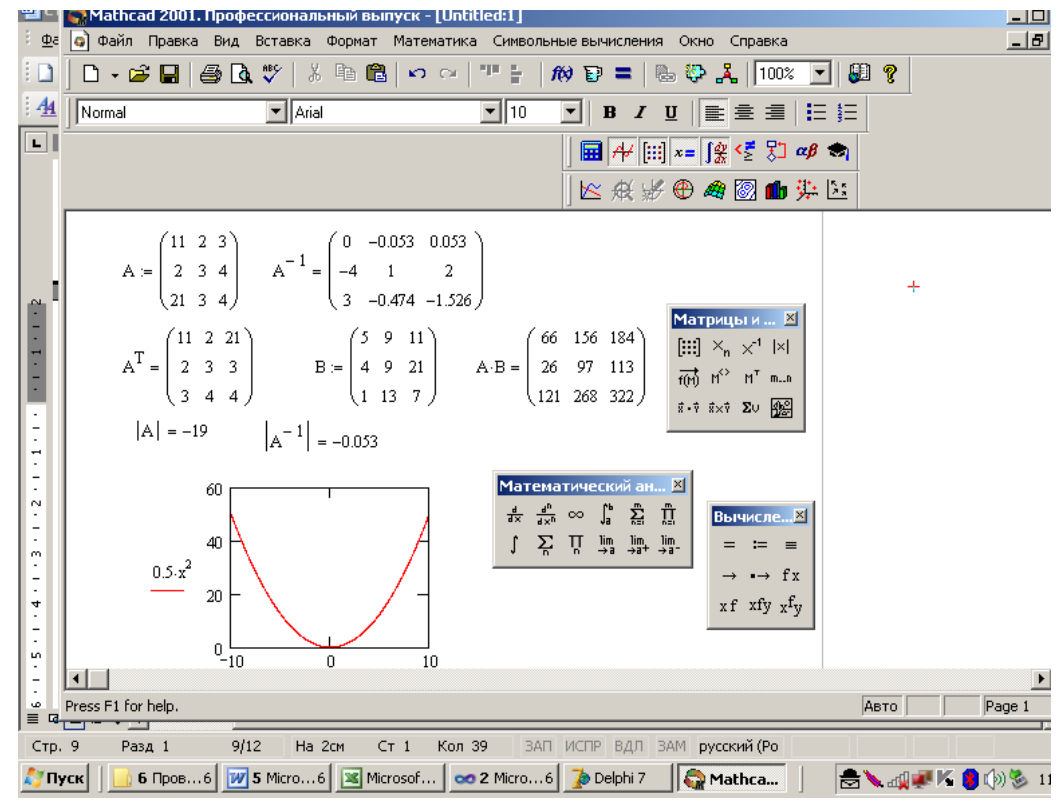

Fig. 3. Use of MathCad environment as a tool for mathematical knowledge formation.

As was stated above, the use of mathematical packages in the problems of matrix algebra, mathematical analysis, computational mathematics, mathematical logic for studying the behavior of functions describing the problematics of professional activity, contributes not only to understanding the logic of methods and operations, but also to formation of mathematical memory, computing culture or culture of the use of mathematical packages in professional tasks. Thus, the sequence of studying the subjects on the intersubject basis offered by us is as follows:

- problems of professional activity, which solution requires the use of mathematics instruments;

- learning the instruments with relevant explanations of a teacher;

- problem solution and analysis;

- study of mathematics section invariant.

Moreover, it is necessary to give more self-study tasks for the last step. (For example, analyze the "Matrix methods for solving the systems of linear equations"). Prepare the presentation and report for the workshop. Highlight the problem classes from the professional field, which solutions are based on knowledge from this section in the report).

Mathematical packages are very comfortable in organization of educational research experiments in obtaining the various states of objects from professional activity when changing the parameters in the mathematical model. Such classes of research nature 
contribute to formation of mathematical thinking, mathematical memory thanks to the ability of visualizing the calculations and their results.

We would like to note that even the lack of highly professional and expensive mathematical software packages is not a problem for familiarity of bachelors of nonmathematical profiles with computational experiments in the problems from professional activity.

Firstly, Microsoft EXCEL electronic worksheet is used in all educational institutions. This software is a unique tool for learning the bases of mathematical logic, probability theory, mathematical statistics, some sections of mathematical analysis, matrix algebra, computational mathematics, bases of mathematic simulation by bachelors of nonmathematical specialties.

Secondly, there are currently many free software products such as EULER, SciLab.

Here is the wording of the certain types of tasks:

Select the tasks from the professional field to be solved via algebra. Calculate the sum, the product of two matrices. Find the inverse matrix. Solve the system of linear equations. Check your solutions in EXCEL (MathCad, EULER, SciLab)).

Select the tasks from the professional field to be solved via the knowledge from the field of mathematical analysis. Calculate derivatives, definite integrals via the methods of rectangles, trapezia, and tangent lines and test your solutions in EXCEL (MathCad, EULER, SciLab).

Solve the problem of linear programming, transportation problem and perform check by means of computer mathematical packages.

Determine the characteristics of random processes and simulate in EXCEL environment (STATISTICS).

Solve the problem of forecasting the change of any process from your professional activity (cold-related diseases, dynamics of growth in the number of applicants, increase in the amount of cars being bought by population of your city, dynamics of population growth in your city, region) and analyze your models and solutions via the EXCEL tools of statistical functions, Statistics.

Construct various predicates using basic simple functions. Check the validity of your transformational changes in EXCEL.

Simulate various games from the professional field using all function groups.

\section{Results}

There was a need to research in practice how to combine the instruments of mathematical calculations and mathematics invariant in order to achieve the stated objective. It was decided to teach the following intersubject academic disciplines:

- Mathematical statistics in EXCEL environment;

- Linear algebra and solving professional problems in MathCad environment;

- Accompanying of pedagogical experiment via the tools of EXCEL environment and others.

Besides, these disciplines were being studied in parallel with the study of mathematics in some groups. But in the groups wherein the study of these subjects was not associated with the study of mathematics, the teacher himself gave the required fundamental knowledge. The main purpose was to determine the change in the attitude toward mathematics as an instrument for solution of job tasks and basically the dynamics of academic progress quality change in mathematics.

The results were unexpected for us. The quality of academic progress with the same sample of students increased up to $80 \%$. Almost $90 \%$ of students noted the need for 
mathematical knowledge. To match these subjects of the Federal State Educational Standard of the Russian Federation (Federal State Educational Standard 3+), the abovementioned courses were organized later as the optional courses, whereby these courses were announced on the different profiles of non-mathematical areas. Interest in these courses is great one. So, 160 students of the third course of the disciplines listed in Table No.1 with the total number of 217 people registered for the discipline "Mathematical Statistics in EXCEL environment".

\section{Discussion}

Using the EULER, SciLab, Free Math, EXCEL free packages offers the teacher with great opportunities for formation of computer-generated calculations culture.

The quality of mathematics education is changing for the better, when we construct the mathematics education via solving the problems from professional field with the use of mathematical computer-generated packages and we are immersing into the mathematics invariant in this process.

Teaching mathematics in secondary schools, specialized secondary educational establishments, higher education institutions through the occupations tasks oriented toward the region professions has shown a right of existence. The problem books focused on the professions in the region were being additionally used by mathematics teachers in school practice for a long time [4]. The experience of using modern computer mathematical packages for solving the problems from professional field is limited in the practice of universities and colleges.

Our experience in the development and implementation of courses on a multidisciplinary basis in the training system of bachelors and masters of non-mathematical disciplines has led us to the personnel problem. It is not always that mathematicians are aware of other subject areas, and experts in the subject area know the mathematics apparatus.

Therefore, in order to fundamentally change the training of subject teachers, i.e., the teachers leading professional disciplines per training programs, broaden their horizons and knowledge in the field of mathematics application in the professional sphere. We think that there is no contradiction in the fact that one discipline will be led by 2-3 teachers. And over the course of time, this problem will be solved itself through the training of new teaching personnel having the mathematical tools (computational science) in their professional activity.

\section{Conclusion}

Change-over to intersubject education sets a monumental challenge before college-level education, i.e. the challenge of forming new academic disciplines on a multidisciplinary basis; moreover, a professional area is a defining guide for formation of academic disciplines here. Engineering and mathematical training programs stay out of our research.

\section{References}

1. K. K. Colin, Open education, 2, 55 (2006)

2. A. A. Samarsky, Bulletin of the Academy of Sciences of the USSR, 5 (1979)

3. S. I. Gorlov, T. B. Kaziakhmedov, Pedagogical Informatics, 4 (2016) 
4. A. V. Abramov, O. B. Episheva, T. B. Kaziakhmedov, et al. Mathematics and other professions in the Tyumen North: Problem book: Handbook for mathematics teachers of secondary schools of the Tyumen Region (Publishing House of the Ural University, Yekaterinburg; Publishing House of the Pedagogical Institute, Nizhnevartovsk, 1993)

5. A. N. Kolmogorov, Mathematics as a science and profession (Chief Editorial Board of Physical and Mathematical Literature, Nauka, Moscow, 1988)

6. A. V. Dorofeev, Education and Science, 2 (2005)

7. G. Weil Mathematical thinking. Transl. from English and German/Edited by B.V. Biryukov and A.N. Parshin (Chief Editorial Board of Physical and Mathematical Literature, Nauka, Moscow, 1989) 